Research Article

\title{
Nonfragile Estimator Design for Fractional-Order Neural Networks under Event-Triggered Mechanism
}

\author{
Xiaoguang Shao, Ming Lyu $\mathbb{D}^{\mathrm{D}}$, and Jie Zhang \\ School of Automation, Nanjing University of Science and Technology, Nanjing 210094, China \\ Correspondence should be addressed to Ming Lyu; lumtz@163.com
}

Received 25 December 2020; Revised 30 January 2021; Accepted 5 March 2021; Published 29 March 2021

Academic Editor: Wangyan Li

Copyright (c) 2021 Xiaoguang Shao et al. This is an open access article distributed under the Creative Commons Attribution License, which permits unrestricted use, distribution, and reproduction in any medium, provided the original work is properly cited.

\begin{abstract}
This paper is concerned with the nonfragile state estimation for a kind of delayed fractional-order neural network under the event-triggered mechanism (ETM). To reduce the bandwidth occupation of the communication network, the ETM is employed in the sensor-to-estimator channel. Moreover, in order to reflect the reality, the transmission delay is taken into account in the model establishment. Sufficient criteria are supplied to make sure that the augmented system is asymptotically stable by using the fractional-order Lyapunov indirect approach and the linear matrix inequality method. In the end, the theoretical result is shown by means of two numerical examples.
\end{abstract}

\section{Introduction}

The past several decades have witnessed that artificial neural network (ANN) has attracted particular research attention. Because of the outstanding performance, ANN has been extensively applied in image recognition, signal processing [1], fault diagnosis [2], and so on. With the rapid developments of artificial intelligence, the ANN has received considerable attention again by the scholars, which relates to synchronization, dissipativity, attractivity, stability, and state estimation (SE) for various kinds of ANNs [3-7].

As we all know, ANNs are composed of plenty of artificial neurons and the SE problem of the neurons plays a vital role in practical applications. As such, quite a lot of results have been reported on the SE issue (see [8-11] and the reference therein). For the practical systems, the parameter uncertainties are often considered. So far, a lot of research studies regarding uncertain systems have been conducted [12-14]. It is worth noting that the existing results assumed that parameter of the estimator is accurate, which, however, is unrealistic. To solve this problem, we aim to design a nonfragile estimator so as to alleviate the effects induced by the uncertainty of the estimator parameter on the system performance. Till now, some initial results have been published on the nonfragile controller design problems [14-17].

In the networked systems, the network bandwidth is always limited which therefore may result in network congestion when a large amount of data is transmitted. Up to now, the network-induced phenomena including transmission delay, packet loss, and quantification have been discussed adequately. In recent years, much attention has been focused on the ETM and many communication protocols, which aims to avoid the occurrence of the network-induced phenomena. Based on the ETM, plenty of literature has been available on stability analysis, eventtriggered condition design, controller/filter design, and so on [18]. Noting that compared with time-triggered mechanism, the ETM exhibits better performance because the necessary sampling depends on the "event" rather than the "time" $[19,20]$.

In addition, by applying the fractional calculus to the ANNs, the researchers have found that the performance of the fractional-order models is better than integer-order ones, especially in the aspect of memory and hereditary. Till now, some novel fractional-order theories and methods concerning the ANNs have been proposed. For example, a nonfragile nonlinear fractional-order observer is designed in 
[21] and an adaptive event-triggered scheme has been developed in [22]. But these existing fractional-order systems employed ETM are introduced with single delay or without only. Especially, it is a challenge in a fractional system. However, the problem of multiple time delays in real systems is often encountered. Nevertheless, there are few related studies on the nonfragile SE for fractional-order neural network based on ETM with multiple time delays, which motivates us to shorten this gap.

Inspired by the aforementioned lines, a nonfragile state estimator is designed for a class of fractional-order neural networks (FNNs) based on ETM. The advantages in this paper are as follows: (1) compared with the existing estimators, a fractional-order nonfragile estimator is first constructed; (2) to save bandwidth resources, an ETM is applied in the SE problem of the fractional-order neural network; (3) the LMI method and the fractional Lyapunov indirect method are adopted to design the state estimator.

The remaining content is outlined as follows. In Section 2 , some preliminary knowledge is recalled. In Section 3, state estimation criteria are voiced. In Section 4, two numerical examples are given with some simulation figures to support the theorems.

Notation. Throughout this paper, $Z^{T}$ and the symbol * in matrix $Z$ represent matrix transposition and the symmetric term, respectively. $\mathbb{R}$ is the set of integers, and $\mathbb{R}^{n}$ denotes the $n$-dimensional Euclidean space. $I_{n}$ means $n$-dimensional identity matrix. $\mathscr{P}>0(\mathscr{P}<0)$ is defined as a positive-definite (negative-definite) matrix. $\|z\|$ is the Euclidean norm of a vector $z$ in $R_{n} \cdot \lambda_{\max }(R)\left(\lambda_{\min }(R)\right)$ represents the maximum (minimum) eigenvalue of $R$ and $\operatorname{sym}(Y)$ means $Y+Y^{T}$.

\section{Preliminaries and Problem Formulation}

Some fractional definitions and model descriptions are presented firstly. In addition, some important lemmas that will be used in Section 3 are also presented.

Definition 1 (see $[23,24]$ ). For $h(t)$, the fractional integral form is defined as

$$
I^{\alpha} h(q)=\frac{1}{\Gamma(\alpha)} \int_{q_{0}}^{q}(q-\theta)^{\alpha-1} f(\theta) \mathrm{d} \theta, \quad \alpha \in \mathbb{R}^{+},
$$

where $q \geq q_{0}$ and $\Gamma(\cdot)$ is a gamma function.

Definition 2 (see $[23,24]$ ). Caputo's derivative of $h(q)$ is denoted by

$$
{ }_{q_{0}}^{C} D_{q}^{\alpha} h(q)=\frac{1}{\Gamma(z-\alpha)} \int_{q_{0}}^{q}(q-\theta)^{z-\alpha-1} h^{(z)}(\theta) \mathrm{d} \theta, \quad \alpha \in(z-1, z), h(t) \in \iota^{z}\left(\left[t_{0}, \infty\right), \mathbb{R}\right),
$$

where $q \geq q_{0}$ and $z$ is a positive integer.

In what follows, $D^{\alpha}$ stands for ${ }_{q_{0}}^{C} D_{q}^{\alpha}$ for the convenience of presentation. In this paper, let us consider the following FNN model:

$$
\left\{\begin{array}{l}
D^{\alpha} v(t)=-\mathscr{C} v(t)+\mathscr{A} \hbar(v(t))+\mathscr{V} \\
y(t)=D v(t)
\end{array}\right.
$$

where $\alpha \in(0,1)$ is the predetermined fractional order, $\mathscr{C}=$ $\operatorname{diag}\left\{c_{1}, c_{2}, \ldots, c_{n}\right\}$ with $c_{i}>0(i=1,2, \ldots, n), \mathscr{A}=\left(a_{i j}\right)_{n \times n}$ is the connection matrix, the vector $v(t)=\left(v_{1}(t), v_{2}(t), \ldots, v_{n}(t)\right)^{T} \in \mathbb{R}^{n}$ stands for the neuron state, $\hbar(v(t))=\left(\hbar_{1}\left(v_{1}\right), \hbar_{2}\left(v_{2}\right), \ldots, \hbar_{n}\left(v_{n}\right)\right)^{T}$ denotes the activation function of the neurons, $y(t)$ is the measurement output, $\mathscr{V}$ is the system input, and $D$ is a known constant matrix.

In what follows, ETM is introduced in order to reduce the communication burden. The event-triggered condition is predesigned as follows:

$$
e_{y}^{T}(t) e_{y}(t) \leq \sigma y^{T}\left(t_{k} h+j h\right) y^{T}\left(t_{k} h+j h\right),
$$

where $e_{y}(t)=y\left(t_{k} h+j h\right)-y\left(t_{k} h\right), \sigma$ is a given constant, $j h$ and $t_{k} h$ are the sampling instant and the release instant, respectively, $y\left(t_{k} h+j h\right)$ stands for the latest sampled signal, and $n_{i} h=t_{k+1} h-t_{k} h$ denotes the release period.
Remark 1. The sensor is time-driven at discrete instants, which can avoid the Zeno behavior. Moreover, when $\sigma=0$, ETM becomes a time-triggered one.

In this paper, the transmission delay $d_{k} \in[0, \bar{d})$ between sensor and estimator is considered, where $\bar{d}$ is a positive scalar. Therefore, $t_{k} h+d_{k}$ is the arrival time of the transmitted data from sensor to estimator.

In view of [25], the holding interval can be rewritten as $\left[t_{k} h+d_{k}, t_{k+1} h+d_{k+1}\right) \cup \underset{j=0}{j=d_{M}} I_{j}$, where $I_{j}=\left[t_{k} h+j h+\bar{d}\right.$, $\left.t_{k} h+j h+h+\bar{d}\right)$. For the convenience of analysis, denote $d(t)=t-t_{k} h-j h$, and then we have $0 \leq d(t) \leq h+\bar{d} \triangleq d_{M}$. Then, the measurement outputs arrived at the estimator can be rewritten as

$$
\bar{y}(t)=e_{y}(t)+y(t-d(t))=D e_{k}(t)+y(t-d(t)),
$$

where $e_{k}(t)$ is the error vector.

Design a nonfragile state estimator for system (3) as follows:

$$
\left.D^{\alpha} \widehat{v}(t)=-\mathscr{C} \widehat{v}(t)+\mathscr{A} \widetilde{\hbar}(\widehat{v}(t))+\mathscr{V}+(K+\Delta K)[\bar{y}(t)-D \widehat{v})\right],
$$

where $\widehat{v}(t) \in \mathbb{R}^{n}$ stands for the estimate of $v(t), K \in \mathbb{R}^{n \times q}$ is the gain matrix to be determined, and $\Delta K$ represents the gain variation that satisfies $\Delta K=M \mathscr{F}(t) N$, in which $M$ and 
$N$ are known real matrices and $\mathscr{F}(t)$ is an unknown satisfying $\mathscr{F}^{T}(t) \mathscr{F}(t) \leq I$.

Defining $e(t)=v(t)-\widehat{v}(t)$, the estimation error dynamics can be obtained from (3) and (5) as follows:

$$
\begin{aligned}
D^{\alpha} e(t)= & -[\mathscr{C}-(K+\Delta K) D] e(t)+\mathscr{A} \hbar(e(t)) \\
& -(K+\Delta K) D e_{k}(t) \\
& -(K+\Delta K) D v(t-d(t))+(K+\Delta K) D v(t),
\end{aligned}
$$

where $\hbar(e(t)) \triangleq \widetilde{\hbar}(v(t))-\widetilde{\hbar}(\widehat{v}(t))$.

For notation simplicity, we define $\eta(t) \triangleq\left[v^{T}(t) e^{T}(t)\right]^{T}$. An augmented system model from (3) and (8) is given in the following form:

$$
D^{\alpha} \eta(t)=\widetilde{C} \eta(t)+\bar{A} \varphi(\eta(t))+\widetilde{E} e_{k}(t)+\widetilde{K} \eta(t-d(t)),
$$

where

$$
\begin{aligned}
& \bar{C}=\left[\begin{array}{cc}
-C & 0 \\
(K+\Delta K) D & -C-(k+\Delta K) D
\end{array}\right], \bar{A}=\left[\begin{array}{ll}
\mathscr{A} & 0 \\
0 & \mathscr{A}
\end{array}\right], \widetilde{K}=\left[\begin{array}{cc}
0 & 0 \\
-(K+\Delta K) D & 0
\end{array}\right], \\
& \widetilde{E}=\left[\begin{array}{c}
0 \\
-(K+\Delta K) D
\end{array}\right], \varphi(\eta(t)) \triangleq\left[\hbar^{T}(v(t)) \tilde{\hbar}^{T}(e(t))\right]^{T} .
\end{aligned}
$$

Lemma 1 (see [26]). For $\xi_{1}$ and $\xi_{2} \in \mathbb{R}^{n}$ and any positive scale $\epsilon>0$, one has

$$
\psi_{1}^{T} \psi_{2}+\psi_{2}^{T} \psi_{1} \leq \epsilon \psi_{1}^{T} \psi_{1}+\epsilon^{-1} \psi_{2}^{T} \psi_{2}
$$

Lemma 2 (see [27]). For $\forall \alpha \in(0,1)$ and $t \geq 0$, if $v(t) \in \mathbb{R}^{n}$ is continuous and differential, then

$$
D^{\alpha} v^{T}(t) \mathrm{Q} v(t) \leq 2 v^{T}(t) Q D^{\alpha} v(t) .
$$

Lemma 3 (see [28]). For matrices $₫, \mathscr{E}, \mathscr{H}$, where $₫$ is symmetric, the inequality

$$
\emptyset+\mathscr{E} \mathscr{F} \mathscr{H}+(\mathscr{E} \mathscr{F} \mathscr{H})^{T}<0
$$

holds if and only if

$$
\varpi+\xi \mathscr{E} \mathscr{E}^{T}+\xi^{-1} \mathscr{H}^{T} \mathscr{H}<0,
$$

in which $\xi>0$ refers to a scalar and $\mathscr{F}^{T} \mathscr{F}<I$.

Lemma 4 (see [29]). Consider a class of fractional-order nonlinear systems:

$$
D^{\alpha} \eta(t)=h(t, \eta(t-d(t))),
$$

and the initial condition is $\eta\left(t_{0}\right)=\phi \in C\left(\left[t_{0}-\tau, t_{0}\right], \mathbb{R}^{n}\right)$. Suppose that $\omega_{i}(s)(i=1,2): \mathbb{R} \longrightarrow \mathbb{R}$ are positive functions, and $\omega_{1}(0)=\omega_{2}(0)=0, \omega_{2}\left(s_{1}\right)<\omega_{2}\left(s_{2}\right)\left(\forall 0<s_{1}<s_{2}\right)$. If there exist two constants $0<\mu<\epsilon$ and a continuous differential function $V: \mathbb{R} \times \mathbb{R}^{n}$ such taht $\omega_{1} \leq V(t, x) \leq \omega_{2}$ satisfying

$$
\begin{aligned}
D \alpha V(t, \eta(t)) \leq & -\varepsilon V(t, \eta(t)) \\
& +\mu \sup _{-d_{M} \leq-d(t) \leq 0} V(t+d(t), x(t+\eta(t-d(t)))),
\end{aligned}
$$

then the fractional-order system is globally uniformly asymptotically stable.

Lemma 5 (see [30]). The matrix

$$
\phi=\left[\begin{array}{ll}
\phi_{11} & \phi_{12} \\
\phi_{12}^{T} & \phi_{22}
\end{array}\right]<0,
$$

holds if and only if $(a) \phi_{22}<0, \phi_{11}-\phi_{12} \phi_{22}^{-1} \phi_{12}^{T}<0$, or (b) $\phi_{11}<0, \phi_{22}-\phi_{12}^{T} \phi_{11}^{-1} \phi_{12}<0$.

Lemma 6 (see [31]). $V$ is continuous on $\left[t_{0},+\infty\right)$ and bounded on $\left[t_{0}-\rho, t_{0}\right]$. If there exist $p, q$ such that

$$
D_{t}^{\alpha} V(t) \leq-p V(t)+\sum_{k=1}^{m} q_{h} \sup _{-\rho_{h} \leq \omega \leq 0} V(t+\omega), \quad t \geq t_{0},
$$

where $\quad 0<\alpha<1, q_{k}>0, p>\sum_{k=1}^{m} q_{k}$

$\rho=\max \left\{\rho_{1}, \rho_{2}, \ldots, \rho_{m}\right\}$, then $\lim _{t \longrightarrow \infty} V(t)=0$.

and

\section{Main Results}

Theorem 1. For the given positive scalars $\varepsilon>\mu>0$, system (8) is globally asymptotically stable if there exist a symmetric matrix $\mathscr{P}=\operatorname{diag}\left\{\mathscr{P}_{1}, \mathscr{P}_{2}\right\}>0$ and four scalars $\beta_{i}(i=1,2,3)>0, \gamma>0$ satisfying the following $L M I$ :

$$
\begin{gathered}
\Phi=\left[\begin{array}{cccc}
\Phi_{11} & \Phi_{12} & \Phi_{13} & \Phi_{14} \\
* & \Phi_{22} & 0 & 0 \\
* & * & \Phi_{33} & 0 \\
* & * & * & \Phi_{44}
\end{array}\right]<0, \\
\beta_{2} \sigma \widetilde{I}^{T} \widetilde{I}+\beta_{3} \bar{I}_{1}^{T} \bar{I}_{1}-\mu \mathscr{P}<0,
\end{gathered}
$$


where

$$
\begin{aligned}
\Phi_{11} & =\left[\begin{array}{cc}
\Lambda_{11} & D^{T} X^{T}-\gamma D^{T} N^{T} N D \\
* & \Lambda_{2}
\end{array}\right], \Phi_{12}=\left[\begin{array}{cc}
\mathscr{P}_{1} \mathscr{A} & 0 \\
0 & \mathscr{P}_{2} \mathscr{A}
\end{array}\right], \Phi_{14}=\left[\begin{array}{cc}
0 & 0 \\
0 & \mathscr{P}_{2} \bar{M}
\end{array}\right], \\
\Phi_{13} & =\left[\begin{array}{cc}
-\gamma D^{T} N^{T} N D & -\gamma D^{T} N^{T} N D \\
-X D-\gamma D^{T} N^{T} N D & -X D-\gamma D^{T} N^{T} N D
\end{array}\right], \Phi_{22}=\left[\begin{array}{cc}
-\beta_{1} \bar{I}_{1} & 0 \\
0 & -\beta_{1} \bar{I}_{1}
\end{array}\right], \\
\Phi_{33} & =\left[\begin{array}{cc}
-\beta_{2} \bar{I}_{1}+\gamma D^{T} N^{T} N D & \gamma D^{T} N^{T} N D \\
* & -\beta_{3} \bar{I}_{1}+\gamma D^{T} N^{T} N D
\end{array}\right], \Phi_{44}=\left[\begin{array}{cc}
-\beta_{3} \bar{I}_{1} & 0 \\
0 & -\gamma \bar{I}_{1}
\end{array}\right], \\
\Lambda_{11} & =-\mathscr{P}_{1} C-C_{1}^{T} \mathscr{P}+\beta_{1} M_{11}^{T} M_{11}+\epsilon \mathscr{P}_{1}+\gamma D^{T} N^{T} N D, \bar{I}_{1}=\operatorname{diag}\{I, I\}, \\
\Lambda_{22} & =\operatorname{sym}\left\{-\mathscr{P}_{2} C-X D\right\}+\beta_{1} M_{12}^{T} M_{12}+\epsilon \mathscr{P}_{2}+\gamma D^{T} N^{T} N D .
\end{aligned}
$$

Furthermore, the nonfragile estimator gain $K$ of (8) is designed as $K=\mathscr{P}_{2}^{-1} X$.

$$
\widetilde{C}=\left[\begin{array}{cc}
-C & 0 \\
K D & -C-K D
\end{array}\right], \bar{E}=\left[\begin{array}{c}
0 \\
-K D
\end{array}\right], \mathscr{P}=\left[\begin{array}{cc}
\mathscr{P}_{1} & 0 \\
0 & \mathscr{P}_{2}
\end{array}\right], \bar{K}=\left[\begin{array}{cc}
0 & 0 \\
-K D & 0
\end{array}\right] .
$$

Considering system (8), design the following Lyapunov function:

$$
V(t)=\eta^{T}(t) \mathscr{P} \eta(t) .
$$

From Lemma 2, one obtains

$$
\begin{aligned}
D^{\alpha} V(t) \leq & 2 \eta^{T}(t) \mathscr{P} D^{\alpha} \eta(t) \\
= & 2 \eta^{T}(t) \mathscr{P}[\widetilde{C} \eta(t)+\bar{A} \varphi(\eta(t)) \\
& \left.+\widetilde{E} e_{k}(t)+\widetilde{K} \eta(t-d)\right] .
\end{aligned}
$$

It follows from Lemma 3 that

$$
\begin{aligned}
& 2 \eta^{T}(t) \mathscr{P} \bar{A} \varphi(\eta(t)) \leq \beta_{1}^{-1} \eta^{T}(t) \mathscr{P} \overline{A A}^{T} \mathscr{P} \eta(t) \\
&+\beta_{1} \eta^{T}(t) G^{T} G \eta(t), \\
& 2 \eta^{T}(t) \mathscr{P} \widetilde{E} e_{k}(t) \leq \beta_{2}^{-1} \eta^{T}(t) \mathscr{P} \bar{K} \widetilde{E}^{T} \mathscr{P} \eta(t)+\beta_{2} e_{k}^{T}(t) e_{k}(t) \leq \beta_{2}^{-1} \eta^{T}(t) \mathscr{P} \widetilde{E} \widetilde{E}^{T} \mathscr{P} \eta(t)+ \\
& \beta_{2} \sigma \eta^{T}(t-d(t)) \widetilde{I}^{T} \widetilde{I} \eta(t-d(t)), \\
& 2 \eta^{T}(t) \mathscr{P} \bar{K} \eta(t-d(t)) \leq \beta_{3}^{-1} \eta^{T}(t) \mathscr{P} \overline{K K}^{T} \mathscr{P} \eta(t) \\
&+\beta_{3} \eta^{T}(t-d(t)) \eta(t-d(t)),
\end{aligned}
$$

where $\bar{I}=[-I, I]$ and $\widetilde{I}=[I, 0]$.

Combining (24)-(36), we can get

$$
\begin{aligned}
D^{\alpha} V(t) \leq & \eta^{T}(t)\left[\mathscr{P} \widetilde{C}+\mathscr{P} \widetilde{E}_{1}+\widetilde{C}^{T} \mathscr{P}+\widetilde{E}_{1}^{T} \mathscr{P}+\beta_{1}^{-1} \mathscr{P} \overline{A A}^{T} \mathscr{P}\right. \\
& \left.+\beta_{1} G^{T} G+\beta_{2}^{-1} \mathscr{P} \widetilde{E} \widetilde{E}^{T} \mathscr{P}+\beta_{3}^{-1} \mathscr{P} \widetilde{K} \widetilde{K}^{T} \mathscr{P}\right] \eta(t) \\
& +\eta^{T}(t-d(t))\left[\beta_{2} \sigma \widetilde{I}^{T} \widetilde{I}+\beta_{3} \bar{I}_{1}^{T} \bar{I}_{1}\right] \eta(t-d(t)) .
\end{aligned}
$$

By employing Lemma 5, $\Phi<0$ is equivalent to

$$
\bar{\Phi}+\gamma \bar{N}^{T} \bar{N}+\gamma^{-1} \overline{M M}^{T}<0,
$$

where

$$
\begin{aligned}
\bar{\Phi} & =\left[\begin{array}{cccc}
\mathscr{P} \bar{C}+\bar{C}^{T} \mathscr{P}+\beta_{1} M_{1}^{T} M_{1}+\varepsilon \mathscr{P}_{1} & \mathscr{P} \bar{A} & \mathscr{P} \bar{E} & \mathscr{P} \overline{E I} \\
* & -\beta_{1} \bar{I}_{1} & 0 & 0 \\
* & * & -\beta_{2} \bar{I}_{1} & 0 \\
* & * & * & -\beta_{3} \bar{I}_{1}
\end{array}\right], \\
\bar{M}^{T} & \left.=\left[0, M^{T} \mathscr{P}_{2}, 0_{n \times 5 n}\right]^{T}\right], \bar{N}=[N D,-N D, 0,0,-N D,-N D, 0] .
\end{aligned}
$$

Defining an equation as follows:

$$
\Delta \Phi=\bar{M} F(t) \bar{N}+\bar{N}^{T} F^{T}(t) \bar{M}^{T},
$$

we have

$$
\Delta \Phi \leq \gamma^{-1} \overline{M M}^{T}+\gamma \bar{N}^{T} \bar{N} .
$$

Furthermore, from (28) and (31), we arrive at $\bar{\Phi}+\Delta \Phi<0$.

Based on Lemma 3, we can obtain 


$$
\begin{aligned}
\mathscr{P} \widetilde{C} & +\mathscr{P} \widetilde{E}_{1}+\widetilde{C}^{T} \mathscr{P}+\widetilde{E}_{1}^{T} \mathscr{P}+\beta_{1}^{-1} \mathscr{P} \overline{A A}^{T} \mathscr{P}+\beta_{1} G^{T} G \\
& +\beta_{2}^{-1} \mathscr{P} \widetilde{E} \widetilde{E}^{T} \mathscr{P}+\beta_{3}^{-1} \mathscr{P} \widetilde{K} \widetilde{K}^{T} \mathscr{P}<-\varepsilon \mathscr{P} .
\end{aligned}
$$

Combining (27) and (32), we have

$$
\begin{aligned}
D^{\alpha} V(t) \leq & -\epsilon \eta^{T}(t) \mathscr{P} \eta(t) \\
& +\mu \sup _{0 \leq d(t) \leq d_{M}} \eta^{T}(t-d(t)) \mathscr{P} \eta(t-d(t)) \\
= & -\varepsilon V(t)+\mu \sup _{0 \leq d(t) \leq d_{M}} V(t-d(t)) .
\end{aligned}
$$

It follows from Lemma 4 that (4) is an asymptotical estimator and system (3) is globally asymptotically stable. The proof is completed.

It is worth noting that the parameter uncertainties are often unavoidable resulting from the inaccuracy of modeling or the changing environment. In addition, the network output is composed of linear and nonlinear parts. Therefore, the following model of FNN is established:

$$
\left\{\begin{array}{l}
D^{\alpha} v(t)=-(C+\Delta C) v(t)+(A+\Delta A) f(v(t))+(B+\Delta B) f(v(t-\tau(t)))+J, \\
y(t)=D v(t)+\tilde{g}(v(t))
\end{array}\right.
$$

where $\tau(t)$ is a time-varying delay satisfying $0 \leq \tau(t) \leq \tau_{M}$. Here, $\tau_{M}$ is a constant. $\Delta A, \Delta B, \Delta C$ are parameter uncertainties which satisfy the following condition:

$$
\left[\begin{array}{lll}
\Delta A & \Delta B & \Delta C
\end{array}\right]=\overline{M_{2}} F_{1}(t)\left[\begin{array}{lll}
N_{1} & N_{2} & N_{3}
\end{array}\right],
$$

where $\bar{M}_{2}, N_{1}, N_{2}, N_{3}$ are known matrices and $F_{1}(t)$ is an unknown matrix function which satisfies $F_{1}(t)^{T} F_{1}(t) \leq I$.
Assumption 1. $\tilde{g}: \mathbb{R}^{n} \longrightarrow \mathbb{R}^{q}$ stands for the nonlinear disturbance which satisfies the Lipschitz condition:

$$
|\widetilde{g}(t, a)-\widetilde{g}(t, b)| \leq|F(a-b)|,
$$

where $\widetilde{g}(t, 0)=0$ and $F$ is a known constant matrix.

The estimator and estimation error dynamics are obtained as follows:

$$
\left\{\begin{aligned}
D^{\alpha} \widehat{v}(t)= & -(C+\Delta C) \widehat{v}(t)+(A+\Delta A) f(\widehat{v}(t))+(B+\Delta B) f(\widehat{x}(t-\tau(t)))+J+(K+\Delta K)(\overline{y(t)}-\overline{D v(t)}), \\
D^{\alpha} e(t)= & -C-(K+\Delta K) D] e(t)+[-\Delta C+\Delta K) D] v(t)+A \tilde{f}(e(t))+\Delta A f(v(t)+B \tilde{f}(e(t-\tau(t))) \\
& +\Delta B f(x(t-\tau(t)))-(K+\Delta K) e_{y}(t)-(K+\Delta K) D x(t-d(t))-(K+\Delta K) g(x(t-d(t))) .
\end{aligned}\right.
$$

The augmented system is derived as follows:

$$
\begin{aligned}
D^{\alpha} \eta(t)= & \widetilde{C} \eta(t)+\widetilde{A} \varphi(\eta(t))+\widetilde{B} \varphi(\eta(t-\tau(t))) \\
& +\widetilde{H} \eta(t-d(t))-\widetilde{G} g(\bar{I} \eta(t-d(t)))-\widetilde{L} e_{y}(t),
\end{aligned}
$$

where

$$
\begin{aligned}
\widetilde{C} & =\left[\begin{array}{cc}
-C-\Delta C & 0 \\
-\Delta C+(K+\Delta K) D & -C-(K+\Delta K) D
\end{array}\right], \widetilde{A}=\left[\begin{array}{cc}
A+\Delta A & 0 \\
\Delta A & A
\end{array}\right], \\
\widetilde{B} & =\left[\begin{array}{cc}
B+\Delta B & 0 \\
\Delta B & B
\end{array}\right], \widetilde{H}=\left[\begin{array}{cr}
0 & 0 \\
-(K+\Delta K) D & 0
\end{array}\right], \bar{I}=\left[\begin{array}{ll}
0 & 0 \\
I & 0
\end{array}\right], \\
\widetilde{G} & =\left[\begin{array}{cr}
0 & 0 \\
K+\Delta K & 0
\end{array}\right], \widetilde{L}=\left[\begin{array}{c}
0 \\
K+\Delta K
\end{array}\right] .
\end{aligned}
$$

The following theorem is given to ensure the above system is asymptotically stable.
Theorem 2. For given positive scalars $\phi, \vartheta_{1}, \vartheta_{2}$ satisfying $\phi>\vartheta_{1}+\vartheta_{2}$, the augmented system (48) is asymptotically stable if there exist a symmetric matrix $\mathscr{P}=\operatorname{diag}\left\{\mathscr{P}_{1}, \mathscr{P}_{2}\right\}>0$ and seven scalars $\gamma_{i}(i=1,2,3)>0, \beta_{i}(i=1,2, \ldots, 4)>0$ satisfying the following LMI:

$$
\begin{aligned}
\Pi & =\left[\begin{array}{cccccc}
\Pi_{11} & \Pi_{12} & \Pi_{13} & \Pi_{14} & \Pi_{15} & \Pi_{16} \\
* & \Pi_{22} & 0 & \Pi_{24} & \Pi_{25} & 0 \\
* & * & \Pi_{33} & 0 & 0 & 0 \\
* & * & * & \Pi_{44} & \Pi_{45} & 0 \\
* & * & * & * & \Pi_{55} & 0 \\
* & * & * & * & * & \Pi_{66}
\end{array}\right]<0, \\
\Theta & =\left[\begin{array}{ccc}
\Lambda 2 & 0 & \\
* & \beta_{1} I-\vartheta_{1} \mathscr{P}_{2}
\end{array}\right]<0
\end{aligned}
$$




$$
\Phi=\left[\begin{array}{cc}
\beta_{2} M_{11}^{T} M_{11}-\vartheta_{2} \mathscr{P}_{1} & 0 \\
* & \beta_{2} M 12^{T} M_{12}-\vartheta_{2} \mathscr{P}_{2}
\end{array}\right]<0, \quad \text { where }
$$

$$
\begin{aligned}
& \Pi_{11}=\left[\begin{array}{cc}
\Lambda 1 & D^{T} X^{T}-\gamma_{3} D^{T} N_{4}^{T} N_{4} D \\
* & \bar{Q}_{2} 2+\gamma_{3} D^{T} N_{4}^{T} N_{4} D
\end{array}\right], \Pi_{12}=\left[\begin{array}{cc}
-\gamma_{3} D^{T} N_{4}^{T} N_{4} D & \gamma_{2} M_{41}^{T} N_{2}^{T} N_{3} \\
-X D+\gamma_{3} D^{T} N_{4}^{T} N_{4} D & 0
\end{array}\right], \\
& \Pi_{13}=\left[\begin{array}{cc}
\mathscr{P}_{1} B & 0 \\
0 & \mathscr{P}_{2} B
\end{array}\right], \Pi_{14}=\left[\begin{array}{cc}
\gamma_{3} D^{T} N_{4}^{T} N_{4} & 0 \\
X-\gamma_{3} D^{T} N_{4}^{T} N_{4} D & 0
\end{array}\right], \Pi_{66}=\left[\begin{array}{cc}
-\gamma_{2} I & 0 \\
* & -\gamma_{3} I
\end{array}\right], \\
& \Pi_{15}=\left[\begin{array}{cc}
-\gamma_{3} D^{T} N_{4}^{T} N_{4} & -\mathscr{P}_{1} \bar{M}_{2} \\
X-\gamma_{3} D^{T} N_{4}^{T} N_{4} D & -\mathscr{P}_{2} \bar{M}_{2}
\end{array}\right], \Pi_{22}=\left[\begin{array}{cc}
-\beta_{1} I+\gamma_{3} D^{T} N_{4}^{T} N_{4} D & 0 \\
* & -\beta_{1} I
\end{array}\right] \text {, } \\
& \Pi_{16}=\left[\begin{array}{cc}
\mathscr{P}_{1} \bar{M}_{2} & 0 \\
\mathscr{P}_{2} \bar{M}_{2} & \mathscr{P}_{2} \bar{M}_{3}
\end{array}\right], \Pi_{24}=\left[\begin{array}{cc}
-\gamma_{3} D^{T} N_{4}^{T} N_{4} & 0 \\
0 & 0
\end{array}\right] \text {, } \\
& \Pi_{45}=\left[\begin{array}{cc}
\gamma_{3} N_{4}^{T} N_{4} & 0 \\
0 & 0
\end{array}\right], \Pi_{33}=\left[\begin{array}{cc}
-\beta_{2} I+\gamma_{2} N_{3}^{T} N_{3} & 0 \\
* & -\beta_{2} I
\end{array}\right] \text {, } \\
& \Pi_{44}=\left[\begin{array}{cc}
-\beta_{3} I+\gamma_{3} N_{4}^{T} N_{4} & 0 \\
* & -\beta_{3} I
\end{array}\right], \Pi_{25}=\left[\begin{array}{cc}
-\gamma_{3} D^{T} N_{4}^{T} N_{4} & 0 \\
0 & 0
\end{array}\right], \Pi_{55}=\left[\begin{array}{cc}
-\beta_{4} I+\gamma_{3} N_{4}^{T} N_{4} & 0 \\
* & -\gamma_{1} I
\end{array}\right] \text {, } \\
& \Lambda 1=\bar{Q}_{11}+\gamma_{2} M_{41}^{T} N_{2}^{T} N_{2} M_{41}+\gamma_{3} D^{T} N_{4}^{T} N_{4} D \text {, } \\
& \Lambda 2=\beta_{1} I+\beta_{3} M_{22}^{T} M_{22}+\beta_{4} \sigma D^{T} D+\beta_{4} \sigma \operatorname{sym}\left\{D^{T} M_{3}\right\}+\beta_{4} \sigma M_{3}^{T} M_{3}-\vartheta_{2} \mathscr{P}_{2} \text {. } \\
& 2 \eta^{T}(t) \mathscr{P} \tilde{H} \eta(t-d(t)) \leq \beta_{1}^{-1} \eta^{T}(t) \mathscr{P} \tilde{H} \tilde{H}^{T} \mathscr{P} \eta(t) \\
& +\beta_{1} \eta(t-d(t))^{T} \eta(t-d(t))
\end{aligned}
$$

Furthermore, the nonfragile estimator gain $K$ is designed as $K=\mathscr{P}_{2}^{-1} X$.
Proof. Construct the following Lyapunov functional:

$$
V(t)=\eta^{T}(t) \mathscr{P} \eta(t) .
$$

From Lemma 2, the following inequality is obtained:

$$
\begin{aligned}
D^{\alpha} V(t) \leq & 2 \eta^{T}(t) \mathscr{P} D^{\alpha} \eta(t) \\
= & 2 \eta^{T}(t) \mathscr{P}[\widetilde{C} \eta(t)+\widetilde{H} \eta(t-d(t))+\widetilde{A} \varphi(\eta(t)) \\
& \left.+\widetilde{B} \varphi(\eta(t-\tau(t)))-\widetilde{G}_{1} g(\bar{I} \eta(t-d(t)))-\widetilde{L} e_{y}(t)\right] .
\end{aligned}
$$

By using Lemma 1 and Lipschitz condition, one gets

$$
\begin{aligned}
& 2 \eta^{T}(t) \mathscr{P} \widetilde{B} \varphi(\eta(t-\tau(t))) \leq 2 \eta(t)^{T} \mathscr{P} \widetilde{B} M_{1} \eta(t-\tau(t)) \\
& \leq \beta_{2}^{-1} \eta(t) \mathscr{P} \widetilde{B} \widetilde{B}^{T} \mathscr{P} \eta+\beta_{2} \eta^{T}(t-\tau(t)) M_{1}^{T} M_{1} \eta(t-\tau(t)),
\end{aligned}
$$

$$
\begin{aligned}
& -2 \eta^{T}(t) \mathscr{P} \widetilde{G} g(\bar{I} \eta(t-d(t))) \leq \beta_{3}^{-1} \eta^{T}(t) \mathscr{P} \widetilde{G} \widetilde{G}^{T} \mathscr{P} \eta(t) \\
& +\beta_{3} \eta^{T}(t-d(t)) \bar{I}^{T} M_{2}^{T} M_{2} \bar{I} \eta(t-d(t)),
\end{aligned}
$$$$
-2 \eta^{T}(t) \mathscr{P} \tilde{L} e_{y}(t) \leq \beta_{4}^{-1} \eta^{T}(t) \mathscr{P} \tilde{L} \widetilde{L}^{T} \mathscr{P} \eta(t)+\beta_{4} e_{y}(t)^{T} e_{y}(t) .
$$ 
From event-triggered condition (5), we can obtain

$$
\begin{aligned}
e_{y}(t)^{T} e_{y}(t) \leq & \sigma y^{T}\left(t_{k} h+j h\right) y\left(t_{k} h+j h\right) \\
\leq & \sigma y^{T}(t-d(t)) y(t-d(t)) \\
\leq & \sigma \eta^{T}(t-d(t)) \bar{D}^{T} \bar{D} \eta(t-d(t)) \\
& +\sigma \eta^{T}(t-d(t))\left[\bar{D}^{T} M_{3} \widetilde{I}+\sigma \widetilde{I}^{T} M_{3}^{T} \bar{D}\right] \eta(t-d(t)) \\
& +\sigma \eta^{T}(t-d(t)) \widetilde{I}^{T} M_{3}^{T} M_{3} \widetilde{I} \eta(t-d(t)) .
\end{aligned}
$$

Combining (45)-(50), one can get

$$
\begin{aligned}
D^{\alpha} V(t) \leq & 2 \eta^{T}(t) \mathscr{P} D^{\alpha} \eta(t) \\
\leq & \eta^{T}(t)\left[\mathscr{P} \widetilde{C} \eta(t)+\widetilde{C}^{T} \mathscr{P}+\mathscr{P} \widetilde{A} M_{4}+M_{4}^{T} \widetilde{A}^{T} \mathscr{P}\right. \\
& +\beta_{1}^{-1} \mathscr{P} \tilde{H} \widetilde{H}^{T} \mathscr{P}+\beta_{2}^{-1} \mathscr{P} \widetilde{B} \widetilde{B}^{T} \mathscr{P}+\beta_{3}^{-1} \mathscr{P} \widetilde{G} \widetilde{G}^{T} \mathscr{P} \\
& \left.+\beta_{4}^{-1} \mathscr{P} \widetilde{L} \widetilde{L}^{T} \mathscr{P}\right] \eta(t) \\
& +\eta^{T}(t-d(t))\left[\beta_{1} \check{I}+\beta_{3} \bar{I}^{T} M_{2}^{T} M_{2} \bar{I}+\beta_{4} \sigma \bar{D}^{T} M_{3} \widetilde{I}\right. \\
& \left.+\beta_{4} \widetilde{I^{T}} M_{3}^{T} \bar{D}+\widetilde{I}^{T} M_{3}^{T} M_{3} \widetilde{I}\right] \eta(t-d(t)) \\
& \left.\left.+\eta^{T}(t-\tau(t))\right)\left[\beta_{2} M_{1}^{T} M_{1}\right] \eta^{T}(t-\tau(t))\right),
\end{aligned}
$$

and therefore 


$$
\begin{aligned}
Q_{1}= & \operatorname{sym}\left\{\mathscr{P} \widetilde{C}+\mathscr{P} \widetilde{A} M_{4}\right\}+\beta_{1} \mathscr{P} \tilde{H} \tilde{H}^{T} \mathscr{P}+\beta_{2} \mathscr{P} \widetilde{B} \widetilde{B}^{T} \mathscr{P} \\
& +\beta_{1} \mathscr{P} \widetilde{G} \widetilde{G}^{T} \mathscr{P}+\beta_{4} \mathscr{P} \tilde{L} \widetilde{L}^{T} \mathscr{P}+\phi \mathscr{P}, \\
Q_{2}= & \beta_{1} \check{I}+\beta_{3} \bar{I}^{T} M_{2}^{T} M_{2} \bar{I}+\beta_{4} \sigma \bar{D}^{T} \bar{D}+\operatorname{sym}\left\{\beta_{4} \sigma \bar{D}^{T} M_{3} \widetilde{I}\right\} \\
& +\beta_{4} \widetilde{I^{T}} M_{3}^{T} M_{3} \widetilde{I}-\vartheta_{1} \mathscr{P}, \\
Q_{3}= & \beta_{2} M_{1}^{T} M_{1}-\vartheta_{2} \mathscr{P} .
\end{aligned}
$$

By Lemma $5, \Pi<0$ is equivalent to

$$
\left(\overline{\mathrm{Q}}+\gamma_{1} S_{1}^{T} S_{1}+\gamma_{2} S_{2}^{T} S_{2}+\gamma_{3} S_{3}^{T} S_{3}\right)+\gamma_{1}^{-1} R_{1} R_{1}^{T}+\gamma_{2}^{-1} R_{2} R_{2}^{T}+\gamma_{3}^{-1} R_{3} R_{3}^{T}<0,
$$

where

$$
\begin{aligned}
& \overline{\mathrm{Q}}=\left[\begin{array}{ccccc}
\operatorname{sym}\left\{\mathscr{P} C_{1}+\mathscr{P} A_{1} M_{4}\right\} & \mathscr{P} H_{1} & \mathscr{P} B_{1} & \mathscr{P} G_{1} & \mathscr{P} L_{1} \\
* & -\beta_{1} \check{I} & 0 & 0 & 0 \\
* & * & -\beta_{2} \check{I} & 0 & 0 \\
* & * & * & -\beta_{3} \check{I} & 0 \\
* & * & * & * & -\beta_{4} \check{I}
\end{array}\right], \\
& R_{1}=\left[\begin{array}{c}
-\mathscr{P}_{1} \overline{M_{2}} \\
-\mathscr{P}_{2} \overline{M_{2}} \\
0_{7 n \times n}
\end{array}\right], R_{2}=\left[\begin{array}{c}
\mathscr{P}_{1} \overline{M_{2}} \\
\mathscr{P}_{2} \overline{M_{2}} \\
0_{7 n \times n}
\end{array}\right] \text {, } \\
& R_{3}=\left[\begin{array}{c}
0 \\
\mathscr{P}_{2} \overline{M_{2}} \\
0_{7 n \times n}
\end{array}\right], S_{1}=\left[N_{1}, 0_{n \times 8 n}\right] \text {, } \\
& S_{2}=\left[N_{2} M_{41}, 0_{n \times 3 n, N_{3}, 0_{n \times 4 n}}\right], S_{3}=\left[N_{4} D,-N_{4} D,-N_{4} D, 0_{n \times 3 n}, N_{4}, 0, N_{4}\right] .
\end{aligned}
$$

Let

$$
\begin{aligned}
\bar{Q}^{\prime}= & R_{1} F_{1}(t) S_{1}+R_{2} F_{1}(t) S_{2}+R_{3} F_{2}(t) S_{3}+S_{1}^{T} F_{1}^{T}(t) R_{1}^{T} \\
& +S_{2}^{T} F_{1}^{T}(t) R_{2}^{T}+S_{3}^{T} F_{2}^{T}(t) R_{3}^{T} \\
\leq & \gamma_{1} S_{1}^{T} S_{1}+\gamma_{2} S_{2}^{T} S_{2}+\gamma_{3} S_{3}^{T} S_{3}+\gamma_{1}^{-1} R_{1} R_{1}^{T} \\
& +\gamma_{2}^{-1} R_{2} R_{2}^{T}+\gamma_{3}^{-1} R_{3} R_{3}^{T} .
\end{aligned}
$$

Then, one gets $\bar{Q}+\bar{Q}^{\prime}<0$. Based on Lemma 5, (55) and (57) imply that $Q_{1} \leq 0$. According to (40) and (42), we know that $Q_{2}<0$, and $Q_{3}<0$. Therefore, we can obtain

$$
\begin{gathered}
D^{\alpha} V(t)+\phi V(t)-\vartheta_{1} \sup _{-\tau_{M} \leq \omega \leq 0} V(t+\omega, v(t+\omega)) \\
-\vartheta_{2} \sup _{-d_{M} \leq \omega \leq 0} V(t+\omega, v(t+\omega)) \leq 0 .
\end{gathered}
$$

Therefore, system (37) is an asymptotical estimator of (34) by using Lemma 6 . This completes the proof.

\section{Numerical Example}

To illustrate the theoretical results, two numerical examples are shown in this section.

\section{Example 1.}

$$
\mathscr{A}=\left[\begin{array}{ccc}
-1.2 & -0.5 & 1 \\
1 & -0.2 & 1.6 \\
0.2 & 0.8 & -1
\end{array}\right], \mathscr{C}=\left[\begin{array}{ccc}
0.3 & 0 & 0 \\
0 & 0.4 & 0 \\
0 & 0 & 0.3
\end{array}\right], D=\left[\begin{array}{ccc}
0.2 & 0 & 0 \\
0 & 0.28 & 0 \\
0 & 0 & 0.1
\end{array}\right],
$$

where $\bar{M}=\operatorname{diag}\{0.4,0.4,0.4\}, \quad N=\operatorname{diag}\{0.3,-0.3,0.3\}$, and $M_{11}=M_{12}=\operatorname{diag}\{0.2,0.2,0.2\}$.

The other parameters are given as $\alpha=0.98$, $\tau=1, \mathscr{V}=[0.5,0.2,-0.8]^{T}$. The activation function is given as follows:

$$
\left.\hbar\left(v_{1}, v_{2}, v_{3}\right)=\left\{\sin \left(0.4 \pi v_{1}\right), \cos \left(0.2 \pi v_{2}\right), \sin \left(0.6 \pi v_{3}\right)\right)\right\} .
$$

By using Matlab to solve LMIs (26) and (27), the gain matrix $K$ can be obtained as

$$
K=\left[\begin{array}{ccc}
1.5663 & 0.1752 & -1.0655 \\
0.1475 & 1.9113 & -0.8569 \\
-0.7003 & -0.7138 & 1.6980
\end{array}\right]
$$




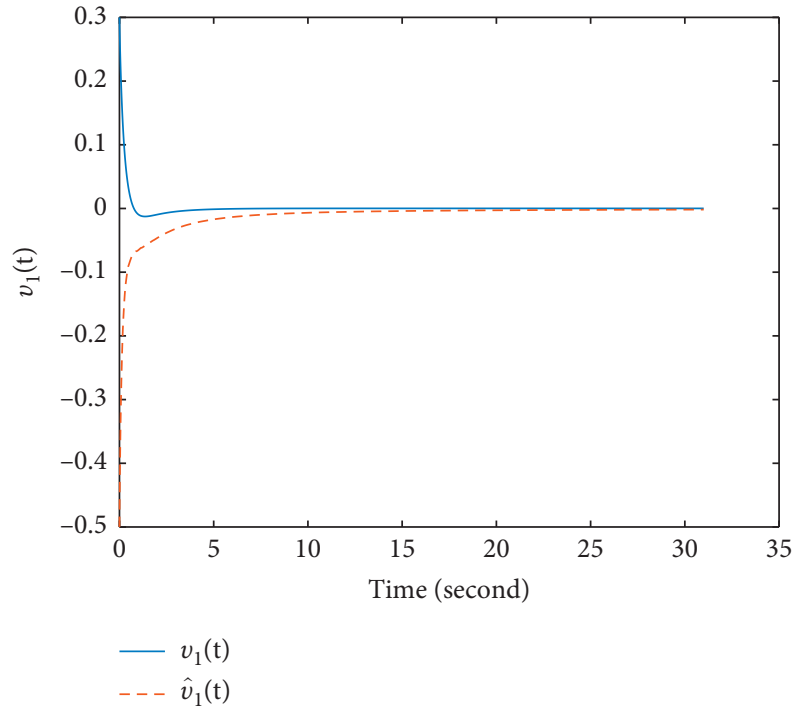

Figure 1: Trajectories of $v_{1}(t)$ and $\widehat{v}_{1}(t)$.

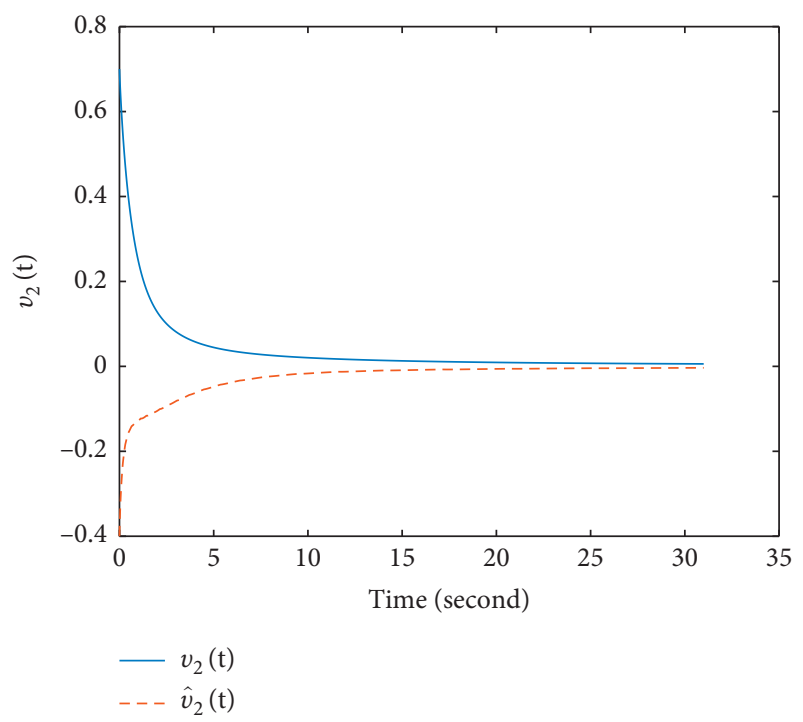

Figure 2: Trajectories of $v_{2}(t)$ and $\widehat{v}_{2}(t)$.

The simulation results are shown in Figures 1-3, where $v_{1}(t), v_{2}(t), v_{3}(t)$ represent the true states and their estimates $\widehat{v}_{1}(t), \widehat{v}_{2}(t), \widehat{v}_{3}(t)$ and the initial conditions are $(\forall t \in[-1,0]): \quad v(t)=[0.3,0.7,-0.3]^{T}, \widehat{v}(t)=[-0.5,-0.4$, $0.2]^{T}$.

Figure 4 shows the estimate error $e_{i}(t) \longrightarrow 0$ as $t \longrightarrow \infty$. According to the simulation results, we can see the effectiveness of the estimator design method. Figure 5 shows the release instants and intervals with the threshold parameter $\sigma=0.06$.

Example 2. To verify that the estimator contains uncertain terms in (34), the following fractional-order model is shown, and the corresponding parameters are as follows:

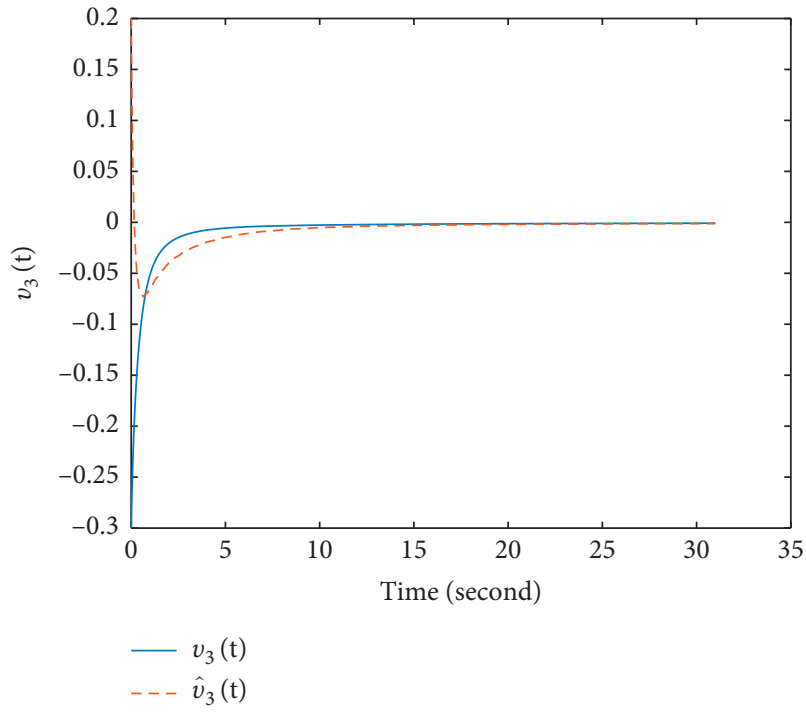

Figure 3: Trajectories of $v_{3}(t)$ and $\widehat{v}_{3}(t)$.

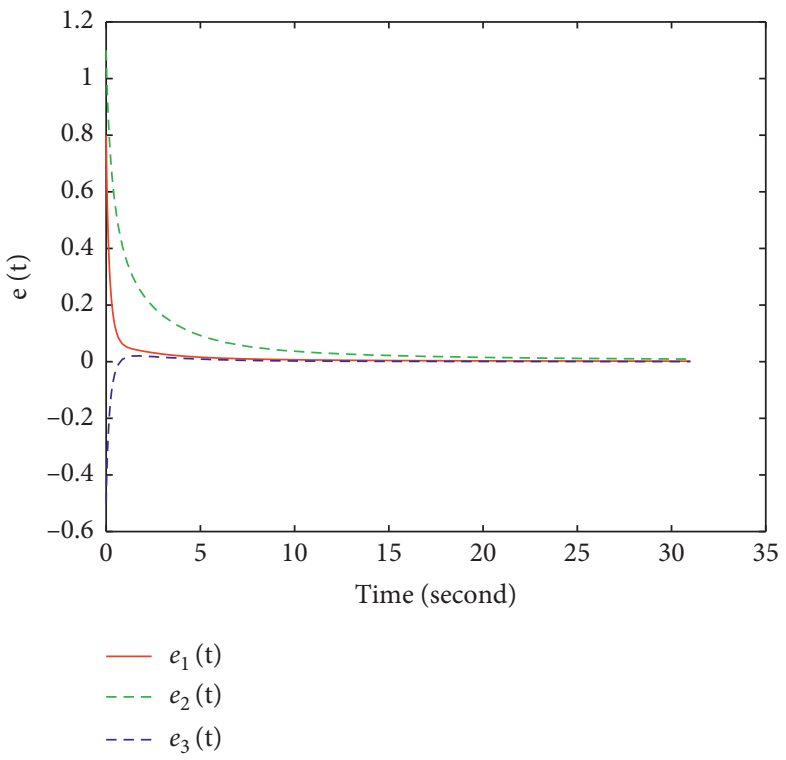

FIGURE 4: The estimation errors.

$A=\left[\begin{array}{ccc}-0.6 & -0.5 & 1 \\ 0.1 & -0.2 & 1 \\ 0.2 . & 0.3 & -1\end{array}\right], B=\left[\begin{array}{ccc}0.2 & -0.2 & 0.1 \\ 0.3 & -0.2 & 0.1 \\ -0.4 & -0.1 & 0.3\end{array}\right], C=\left[\begin{array}{ccc}0.2 & 0 & 0 \\ 0 & 0.2 & 0 \\ 0 & 0 & 0.3\end{array}\right]$,

where $\quad \bar{M}_{2}=\operatorname{diag}\{0.4,0.2,0.4\}, \quad \bar{M}_{3}=\operatorname{diag}\{0.1,0.1,0.1\}$, $M_{11}=M_{12}=M_{41}=M_{42}=\operatorname{diag} \quad\{0.2,0.2,0.2\}$, $N_{1}=N_{2}=\operatorname{diag}\{0.1,0.1,0.1\}$, and $N_{3}=N_{4}=\{0.2,0.2,0.2\}$. Besides, $\alpha=0.92$, and the time delays are set as $\tau_{M}=1, d_{M}=0.1, J=[0.01,0.02,-0.01]^{T}$. The measurement output is $\quad \tilde{g}(t, v)=\left[\tanh \left(0.4 \pi v_{1}\right), \quad \tanh (0.2\right.$ $\left.\left.\left.\pi v_{2}\right), \tanh \left(0.6 \pi v_{3}\right)\right)\right]$. Let the activation function be $\left.\hbar\left(v_{1}, v_{2}, v_{3}\right)=\left[\tanh \left(0.4 \pi v_{1}\right), \tanh \left(0.3 \pi v_{2}\right), \tanh \left(0.2 \pi v_{3}\right)\right)\right]$.

By using Matlab to solve the LMI (37), the gain matrix $K$ can be obtained as 


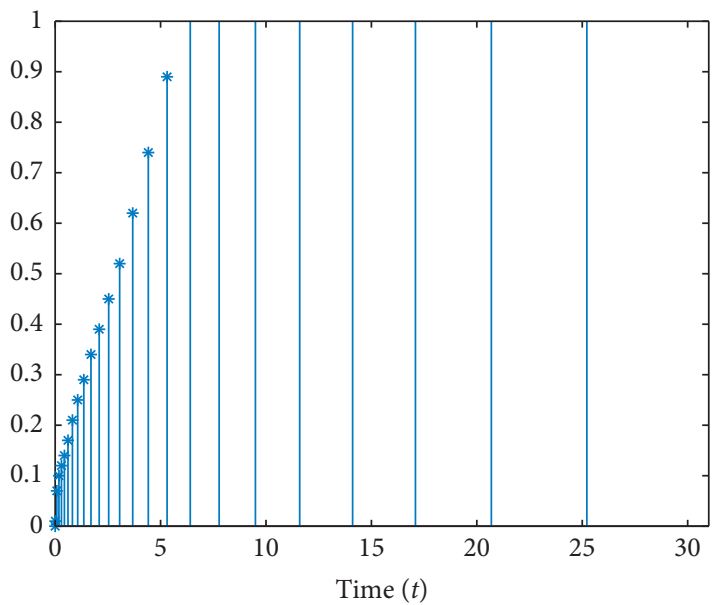

$\rightarrow$ Even-based release instants and release interval

FIgURE 5: Event-triggered release instants and intervals of Theorem 1.

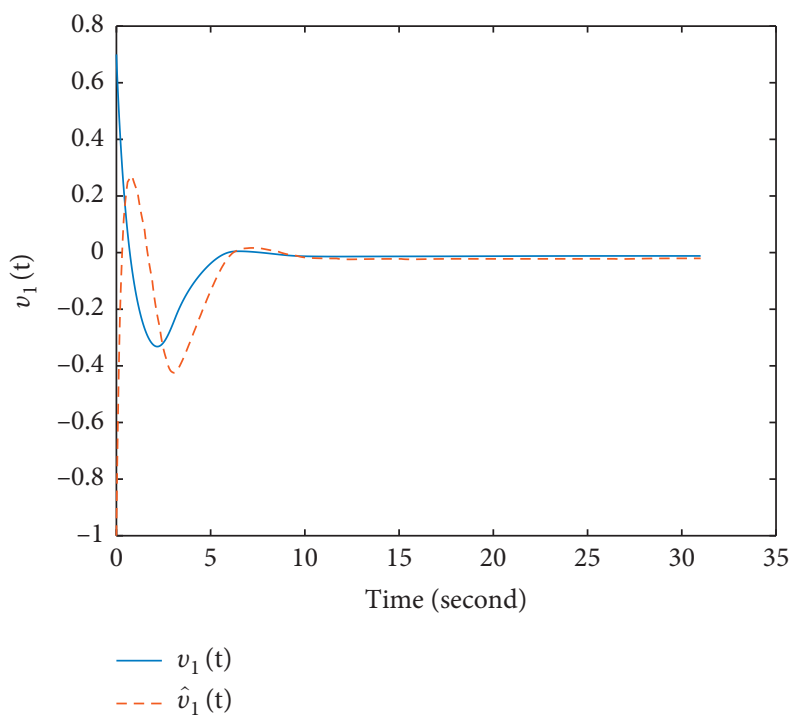

FIgURE 6: Trajectories of $v_{1}(t)$ and $\widehat{v}_{1}(t)$.

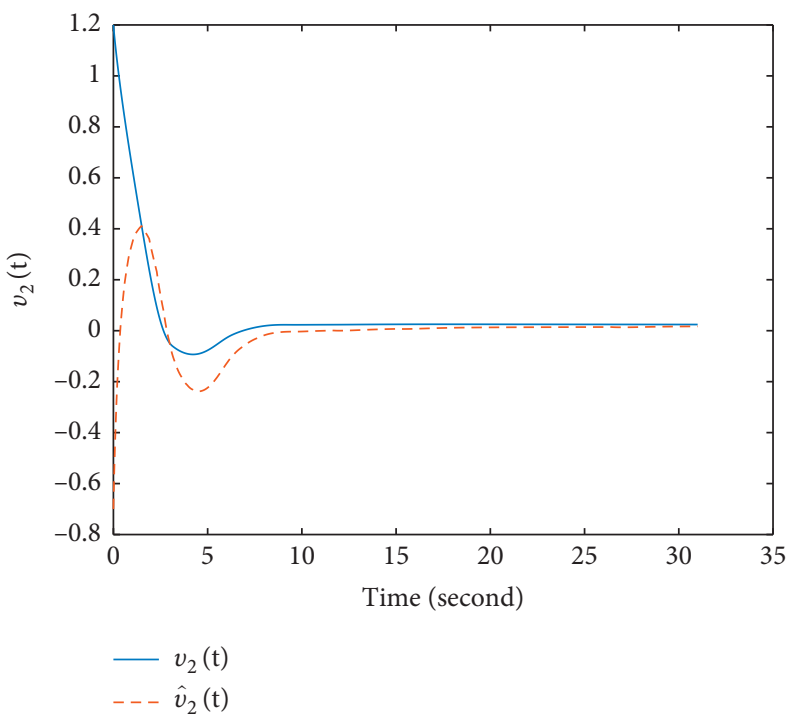

Figure 7: Trajectories of $v_{2}(t)$ and $\widehat{v}_{2}(t)$. 


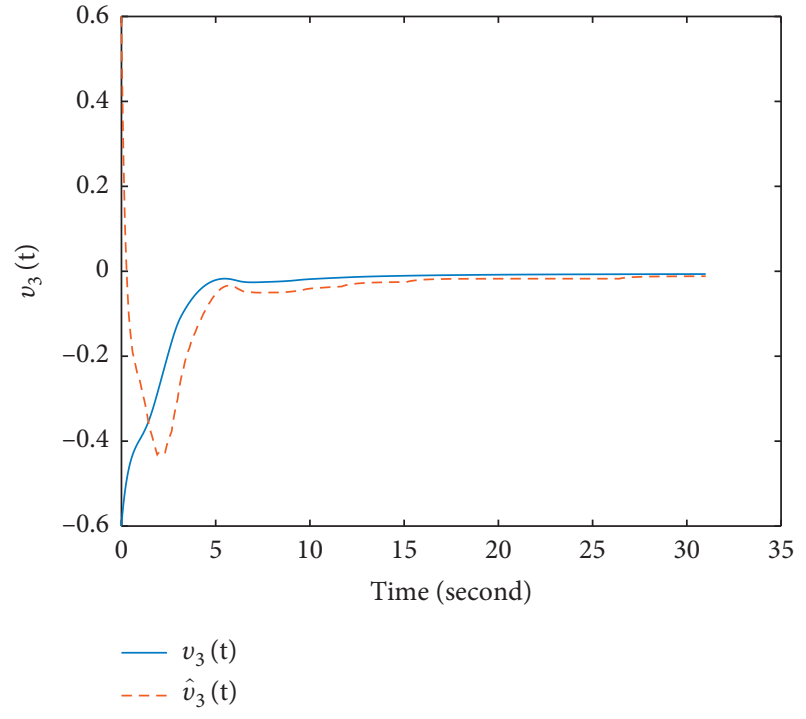

Figure 8: Trajectories of $v_{3}(t)$ and $\widehat{v}_{3}(t)$.

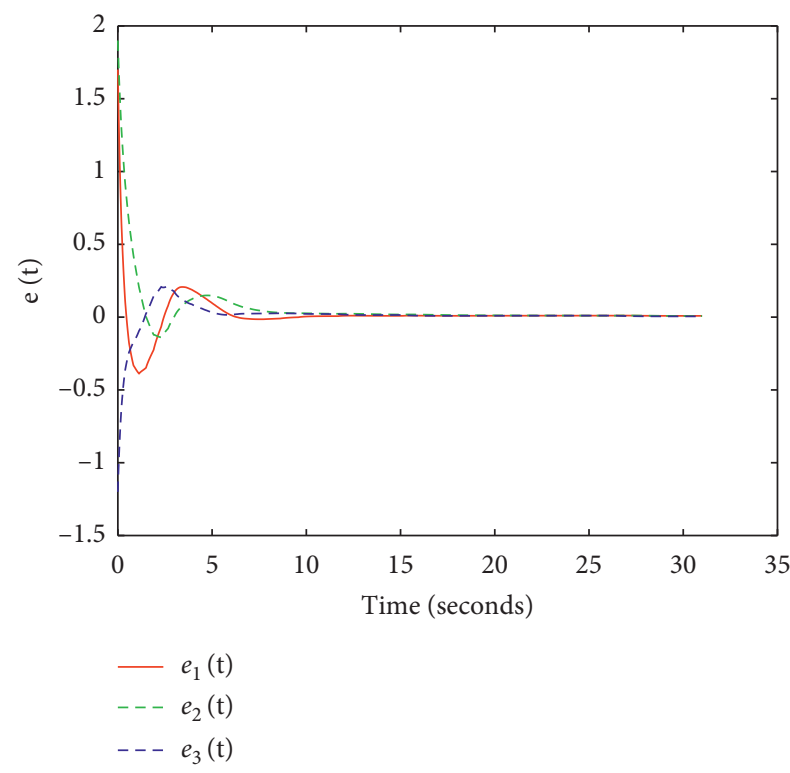

Figure 9: The estimation errors.

$$
K=\left[\begin{array}{ccc}
1.4199 & 0.2566 & -1.4064 \\
0.0808 & 1.2616 & -0.6868 \\
-0.3186 & -0.3723 & 2.8109
\end{array}\right]
$$

The simulation results are shown in Figures 6-8, where $v_{1}(t), v_{2}(t), v_{3}(t)$ represent the true states and their estimates $\widehat{v}_{1}(t), \widehat{v}_{2}(t), \widehat{v}_{3}(t)$ are depicted, respectively, with the initial condition $(\forall t \in[-1,0]):. v(t)=[0.7 ; 1.2 ;-0.6]$, $\widehat{v}(t)=[-1 ;-0.7 ; 0.6]$.

Figure 9 shows the estimate error $e_{1}(t), e_{2}(t), e_{3}(t)$, as $t \longrightarrow \infty$. In Figure 8 , we can see clearly the error states $e_{i}(t) \longrightarrow 0$. Figure 10 shows the release instants and intervals with $\sigma=0.06$. According to the figures, we can see that the simulation results voiced the effectiveness of the estimator design.

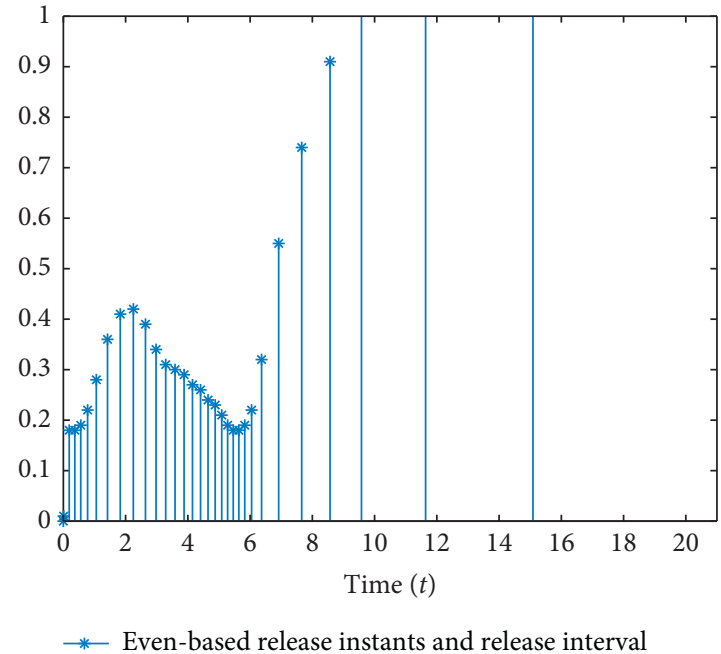

FIGURE 10: Event-triggered release instants and intervals of Theorem 2 .

\section{Conclusions}

This paper has investigated the nonfragile SE issue under the ETM for the FNNs with time delays. Sufficient conditions have been obtained to ensure the asymptotic stability of the considered system by means of the fractional-order Lyapunov functions and the LMI method. The gain matrix of the nonfragile estimator has been characterized by a LMI. At last, two numerical results have confirmed the validity of the designed estimator. In addition, the results could be extended to the SE issue of discrete FNNs with fading measurements and so on.

\section{Data Availability}

In this paper, the initial conditions have been given. As a control system, the data in the Numerical Example is suffienct to support this study.

\section{Conflicts of Interest}

The authors declare that they have no conflicts of interest.

\section{Acknowledgments}

This study was supported by the Natural Science Funds of Jiangsu Province (no. BK20180467).

\section{References}

[1] Y. H. Hu, J. N. Hwang, and S. W. Perry, "Handbook of neural network signal processing," IEEE Transactions on Neural Networks, vol. 16, no. 3, p. 780, 2005.

[2] C. M. Bishop, "Neural networks for pattern recognition," Journal of Scientific Research \& Development Manuscript Pm, vol. 12, no. 5, pp. 1235-1242, 1995.

[3] P. Selvaraj, R. Sakthivel, and O. M. Kwon, "Finite-time synchronization of stochastic coupled neural networks subject to Markovian switching and input saturation," Neural Networks, vol. 105, p. 154, 2018. 
[4] Z. Feng and J. Lam, "Stability and dissipativity analysis of distributed delay cellular neural networks," IEEE Transactions on Neural Networks, vol. 22, no. 6, pp. 976-981, 2011.

[5] Z. Guo, J. Wang, Z. Yan et al., "Attractivity analysis of memristor-based cellular neural networks with time-varying delays," IEEE Transactions on Neural Networks and Learning Systems, vol. 25, no. 4, pp. 704-717, 2014.

[6] Y. Chen, Z. Wang, Y. Liu, and F. E. Alsaadi, "Stochastic stability for distributed delay neural networks via augmented Lyapunov-Krasovskii functionals noise filtering for diagnosing orthopedic implant failures, IEEE Trans," Applied Mathematics and Computation, vol. 338, no. 4, pp. 8691092-8811100, 2018.

[7] Z. Zhao, Z. Wang, L. Zou et al., "Finite-time state estimation for delayed neural networks with redundant delayed channels," IEEE Transactions on Systems, Man, and Cybernetics, vol. 52, pp. 1-11, 2019.

[8] H. Huang, G. Feng, J. Cao et al., "Robust state estimation for uncertain neural networks with time-varying delay," IEEE Transactions on Neural Networks, vol. 19, no. 8, pp. 13291339, 2008.

[9] X.-M. Zhang and Q.-L. Han, "State estimation for static neural networks with time-varying delays based on an improved reciprocally convex inequality," IEEE Transactions on Neural Networks and Learning Systems, vol. 29, no. 4, pp. 1376-1381, 2018.

[10] J. Liang, Z. Wang, X. Liu et al., "State estimation for coupled uncertain stochastic networks with missing measurements and time-varying delays: the discrete-time case," IEEE Transactions on Neural Networks, vol. 20, no. 5, pp. 781-793, 2009.

[11] D. Ding, Q.-L. Han, X. Ge, and J. Wang, "Secure state estimation and control of cyber-physical systems: a survey," IEEE Transactions on Systems, Man, and Cybernetics: Systems, vol. 51, no. 1, pp. 176-190, 2021.

[12] H. Dong, Z. Wang, S. X. Ding, and H. Gao, "Finite-horizon reliable control with randomly occurring uncertainties and nonlinearities subject to output quantization," Automatica, vol. 52, pp. 355-362, 2015.

[13] H. Gao and C. Wang, "A delay-dependent approach to Robust\$H_infty\$Filtering for uncertain discrete-time statedelayed systems," IEEE Transactions on Signal Processing, vol. 52, no. 6, pp. 1631-1640, 2004.

[14] Y. Yu, H. Dong, Z. Wang, W. Ren, and F. E. Alsaadi, "Design of non-fragile state estimators for discrete time-delayed neural networks with parameter uncertainties," Neurocomputing, vol. 182, pp. 18-24, 2016.

[15] J. H. Park, "Robust non-fragile control for uncertain discretedelay large-scale systems with a class of controller gain variations," Applied Mathematics and Computation, vol. 149, no. 1, pp. 147-164, 2004.

[16] F. Yang, H. Dong, Z. Wang, W. Ren, and F. E. Alsaadi, “A new approach to non-fragile state estimation for continuous neural networks with time-delays," Neurocomputing, vol. 197, no. 197, pp. 205-211, 2016.

[17] S. Rajavel, R. Samidurai, J. Cao, A. Alsaedi, and B. Ahmad, "Finite-time non-fragile passivity control for neural networks with time-varying delay," Applied Mathematics and Computation, vol. 297, pp. 145-158, 2017.

[18] D. Ding, Z. Wang, and Q.-L. Han, “A set-membership approach to event-triggered filtering for general nonlinear systems over sensor networks," IEEE Transactions on Automatic Control, vol. 65, no. 4, pp. 1792-1799, 2020.
[19] Z.-P. Jiang and T.-F. Liu, "A survey of recent results in quantized and event-based nonlinear control," International Journal of Automation and Computing, vol. 12, no. 5, pp. 455-466, 2015.

[20] L. Zou, Zi-D. Wang, and D.-H. Zhou, "Event-based control and filtering of networked systems: a survey," International Journal of Automation and Computing, vol. 14, no. 3, 2017.

[21] E. A. Boroujeni and H. R. Momeni, "Non-fragile nonlinear fractional order observer design for a class of nonlinear fractional order systems," Signal Processing, vol. 92, no. 10, pp. 2365-2370, 2012.

[22] M. Xiong, G. Ju, and Y. Tan, "Robust state estimation for fractional-order nonlinear uncertain systems via adaptive event-triggered communication scheme," IEEE Access, vol. 7, pp. 115002-115009, 2019.

[23] I. Podlubny, Fractional Differential Equations: an Introduction to Fractional Derivatives, Fractional Differential Equations, to Methods of their Solution and Some of their applications, Elsevier, Amsterdam, Netherlands, 1998.

[24] A. A. Kilbas, H. M. Srivastava, and J. J. Trujillo, Theory and Applications of Fractional Differential equations, Elsevier, Amsterdam, Netherlands, 2006.

[25] D. Yue, E. Tian, and Q. L. Han, “A delay system method for designing event-triggered controllers of networked control systems," IEEE Transactions on Automatic Control, vol. 58, no. 2, pp. 475-481, 2012.

[26] E. N. Sanchez and J. P. Perez, "Input-to-state stability (ISS) analysis for dynamic neural networks," IEEE Transactions on Circuits and Systems I: Fundamental Theory and Applications, vol. 46, no. 11, pp. 1395-1398, 1999.

[27] S. Zhang, Y. Yu, J. Yu et al., "LMI conditions for global stability of fractional-order neural networks," IEEE Transactions on Neural Networks and Learning Systems, vol. 28, no. 10, pp. 2423-2433, 2017.

[28] L. Xie, "Output feedback Ho control of systems with parameter uncertainty," International Journal of Control, vol. 63, no. 4, pp. 741-750, 1996.

[29] Y. Li, Y. Chen, and I. Podlubny, "Stability of fractional-order nonlinear dynamic systems: Lyapunov direct method and generalized Mittag-Leffler stability," Computers \& Mathematics with Applications, vol. 59, no. 5, pp. 1810-1821, 2010.

[30] S. Boyd, L. El Ghaoui, E. Feron et al., Linear Matrix Inequalities in System and Control Theory, Society for Industrial and Applied Mathematics, Philadelphia, PA, USA, 1994.

[31] P. Liu, M. Kong, and Z. Zeng, "Projective synchronization analysis of fractional-order neural networks with mixed time delays," IEEE Transactions on Cybernetics, 2020. 